\section{Primitive neural stem cells from the mammalian epiblast differentiate to definitive neural stem cells under the control of Notch signaling}

\author{
Seiji Hitoshi, ${ }^{1,2,3,4}$ Raewyn M. Seaberg, ${ }^{2}$ \\ Cheryl Koscik, ${ }^{2}$ Tania Alexson, ${ }^{2}$ \\ Susumu Kusunoki, ${ }^{1}$ Ichiro Kanazawa, ${ }^{1}$ \\ Shoji Tsuji, ${ }^{1}$ and Derek van der Kooy ${ }^{2,5}$ \\ ${ }^{1}$ Department of Neurology, University of Tokyo, Tokyo \\ 113-8655, Japan; ${ }^{2}$ Department of Medical Genetics and \\ Microbiology, University of Toronto, \\ Toronto, Ontario M5S 1A8, Canada
}

Basic fibroblast growth factor (FGF2)-responsive definitive neural stem cells first appear in embryonic day 8.5 (E8.5) mouse embryos, but not in earlier embryos, although neural tissue exists at E7.5. Here, we demonstrate that leukemia inhibitory factor-dependent (but not FGF2-dependent) sphere-forming cells are present in the earlier (E5.5-E7.5) mouse embryo. The resultant clonal sphere cells possess self-renewal capacity and neural multipotentiality, cardinal features of the neural stem cell. However, they also retain some nonneural properties, suggesting that they are the in vivo cells' equivalent of the primitive neural stem cells that form in vitro from embryonic stem cells. The generation of the in vivo primitive neural stem cell was independent of Notch signaling, but the activation of the Notch pathway was important for the transition from the primitive to full definitive neural stem cell properties and for the maintenance of the definitive neural stem cell state.

Received March 31, 2004; revised version accepted May 26, 2004.

The epiblast is endowed with positional information along its anteroposterior axis by the primitive streak stage at embryonic day 6.5 (E6.5), and the anteromedial part of the epiblast is further specified to form neural plate by E7.5 in the mouse embryo. Expression of neural tissue-specific marker genes, such as Nestin and Sox1, first becomes detectable at E7.0-E8.0 (Wood and Episkopou 1999; Kawaguchi et al. 2001). This happens before the first appearance of basic fibroblast growth factor (FGF2)-responsive neural stem cells at E8.5 (Tropepe et al. 1999). Thus, the first neurally specified cell in the epiblast could be a transient neural progenitor cell that is

[Keywords: Neural stem cell; embryonic stem cell; epiblast; neuroectoderm; leukemia inhibitory factor; Notch signaling]

${ }^{3}$ Present address: Division of Neurobiology \& Bioinformatics, National Institute for Physiological Sciences, Aichi 444-8585, Japan.

Corresponding authors.

${ }^{4}$ E-MAIL shitoshi@nips.ac.jp; FAX 81-564-59-5247.

${ }^{5}$ E-MAIL derek.van.der.kooy@utoronto.ca; FAX (416) 978-3844.

Article and publication are at http://www.genesdev.org/cgi/doi/10.1101/ gad.1208404 induced (by signals from mesoderm or endoderm) to yield neural stem cells, or perhaps, more interestingly, a primitive neural stem cell itself that builds the neural plate before giving rise to the definitive neural stem cell. Here, we define the "definitive" neural stem cell as the neural stem cell that is present in the late embryonic or adult brain and proliferates in response to FGF2 (and epidermal growth factor [EGF], or has the potential to acquire EGF responsiveness) to form clonal floating sphere colonies in vitro.

We developed a colony-forming ES sphere assay, in which embryonic stem (ES) cells are cultured in serumfree media in the presence of leukemia inhibitory factor (LIF; at clonal density or as single cells) to generate nes$\mathrm{tin}^{+}$floating sphere colonies (ES spheres; Tropepe et al. 2001). The ES sphere-forming cells show the cardinal features of neural stem cells, self-renewal and neural multipotentiality, but also possess some pluripotency in generating nonneural progeny, and hence were termed primitive neural stem cells (Tropepe et al. 2001). We suggest that in the absence of positive or negative instructive signals, primitive neural stem cells default from ES cells. Although some endogenous signals (e.g., FGF2) may be in fact indispensable for neural induction, survival, or proliferation, these signals may be concealed by autocrine mechanisms (Streit et al. 2000; Tropepe et al. 2001; Wilson and Edlund 2001; Ying et al. 2003). We hypothesize that epiblast cells are inhibited by endogenous factors (e.g., bone morphogenetic proteins [BMPs]) until definitive neural stem cells are created, as this inhibition is relieved when the neural tube forms later in embryogenesis. This hypothesis predicts that primitive neural stem cells can be isolated from the early mouse embryo prior to E7.5 in vivo.

Historically, Notch signaling in Drosophila was thought to maintain cells in an undifferentiated state through a lateral inhibition mechanism (Artavanis-Tsakonas et al. 1995; Kimble and Simpson 1997). The Notch signaling also plays significant roles in mammalian neurogenesis: disruption of Notch pathway genes results in the reduction of the neural stem cell pool size (Nakamura et al. 2000; Hitoshi et al. 2002). The activation of this signaling promotes the symmetrical divisions of neural stem cells, and thereby enhances the self-renewal ability of the neural stem cells. However, little is known about molecular mechanisms underlying the generation of primitive and definitive neural stem cells in vivo. In this study, we used an in vitro colony-forming sphere assay to isolate primitive neural stem cells from early mouse embryos and demonstrated a role for Notch signaling in the generation of definitive neural stem cells.

\section{Results and Discussion}

Mouse E7.5 neuroectoderm was dissociated to single cells and cultured in serum-free media containing LIF to clonally generate nestin ${ }^{+}$floating sphere colonies (Fig. 1A). When dissociated cells from E7.5 CD1 and from green fluorescent protein (GFP)-expressing neuroectoderm were equally mixed (at a final density of 10 cells/ $\mu L)$ and then proliferated to form LIF-dependent spheres, spheres containing both $\mathrm{GFP}^{+}$and $\mathrm{GFP}^{-}$cells never were observed (Fig. 1A), suggesting that the primary LIF-dependent spheres were clonally derived from single cells. 

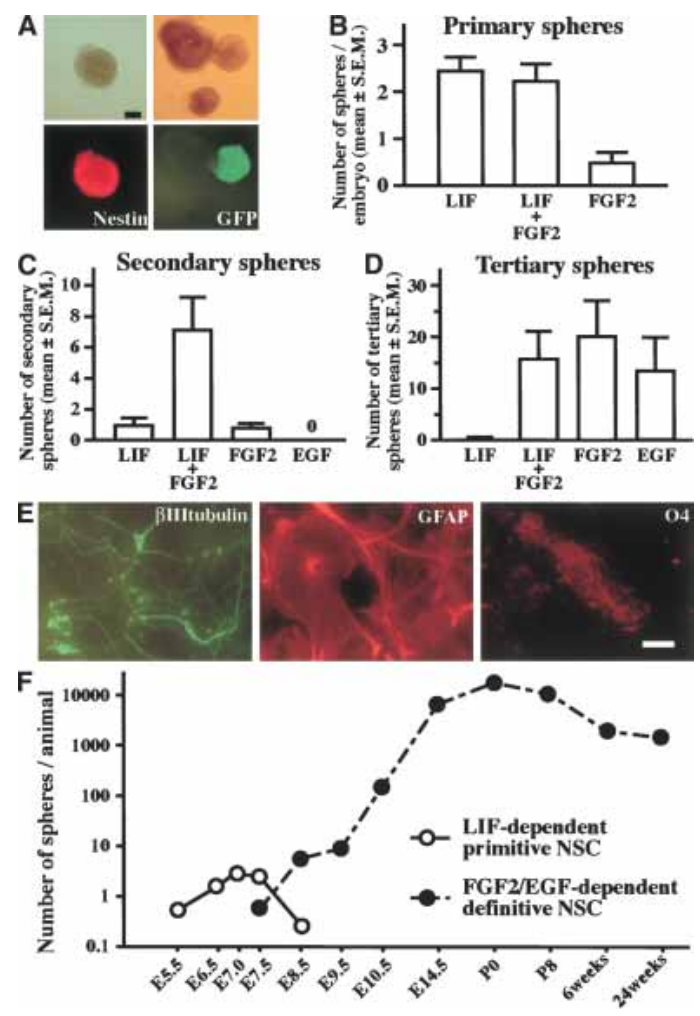

Figure 1. LIF-dependent primitive neural stem cell spheres. $(A)$ The spheres (top left) generated from the E7.5 mouse neuroectoderm were immunopositive for nestin (bottom left). Dissociated E7.5 neuroectoderm cells from CD1 embryos and from GFP mouse embryos were mixed in equivalent proportions (to a final cell density of 10 cells $/ \mathrm{\mu L}$ ) and proliferated to form LIF-dependent spheres (top right). The complete lack of $\mathrm{GFP}^{+}$cells in the white spheres (bottom right) shows that the spheres were clonally derived from single cells. Bar, $0.1 \mathrm{~mm}$. (B) The average sphere numbers in the presence of LIF ( $n=32$ embryos), FGF2 ( $n=16$ embryos), or both ( $n=35$ embryos $)$ per embryo are shown. $(C)$ Single primary LIF-dependent spheres produced secondary spheres in the presence of both LIF and FGF2 $(n=10$ or more). $(D)$ Single secondary spheres cultured in both LIF and FGF2 responded to either FGF2 or EGF to produce tertiary spheres $(n=16$ or more). (E) The primary and passaged E7.5 sphere contained cells that could differentiate into $\beta I I I$ tubulin ${ }^{+}$neurons, $\mathrm{GFAP}^{+}$astrocytes, or $\mathrm{O}^{+}$oligodendrocytes in vitro. Bar, $40 \mu \mathrm{m} .(F)$ The average numbers of LIF-dependent spheres (open circles) and FGF2/EGF-dependent forebrain neurospheres (closed circles) per animal that were isolated at different times throughout development and into the adult mouse are plotted.

At E7.5, very few spheres formed in the media containing only FGF2, and adding FGF2 did not significantly increase the numbers that formed in LIF alone (Fig. 1B). However, FGF2 secreted from the primary LIF-dependent cells themselves (Fig. 2A) may suffice for transducing the FGF2 signal through autocrine or paracrine mechanisms, leaving open the possibility that the activation of FGF signaling is necessary for the formation of spheres from E7.5 neuroectoderm.

Self-renewal and neural multilineage potential are hallmark features of neural stem cells. The primary E7.5 primitive neural stem cell spheres cultured in LIF were capable of producing secondary spheres after dissociation in the presence of both LIF and FGF2, but only very poorly in the presence of LIF alone or FGF2 alone (Fig. 1C). No secondary spheres were found in EGF alone. However, the secondary spheres cultured in both LIF and
FGF2 could be dissociated again to produce clonal tertiary spheres in the presence of either FGF2 alone or EGF alone (Fig. 1D). These neural stem cells then could be maintained and passaged in either of the same media. In vivo, EGF-dependent neural stem cells start to descend from their earlier FGF2-dependent ancestor neural stem cells (Tropepe et al. 1999), and the sequence and time course of their lineage appears to be maintained in vitro by the primitive neural stem cells isolated from the E5.5-E7.5 embryo. Cells from the primary LIF-dependent as well as passaged FGF2- or EGF-dependent sphere could differentiate into neurons, astrocytes, or oligodendrocytes in vitro (Fig. 1E), demonstrating that the LIFdependent primitive neural stem cells (and then their in vitro definitive FGF2- and EGF-dependent neural stem cell progeny) were multipotent in the neural lineage. These observations suggest that the primitive, LIF-dependent neural stem cells derived from E5.5-E7.5 embryos in vivo have a capacity to self-renew and also show neural multipotentiality as definitive neural stem cells.

To exclude the possible involvement of mesenchymal cells in sphere formation, we dissected earlier embryos before the migration of mesodermal tissue beneath the anterior neuroectoderm. LIF-dependent spheres could be isolated from the epiblast at the prestreak stage (E6.5) or even at the egg cylinder stage (E5.5; Fig. 1F). During the E7.5-E8.5 period, LIF-dependent sphere-forming neural stem cells diminished and were replaced by the emergence of FGF2-dependent definitive neural stem cells (Fig. 1F). These results parallel the emergence from ES cells in vitro of LIF-dependent, clonal primitive neural stem cell spheres, which then give rise directly to clonal FGF2-dependent spheres in vitro (Tropepe et al. 2001). Thus, LIF-dependent primitive neural stem cells are present in the epiblast/neuroectoderm of E5.5-E7.5 mouse embryo. These primitive neural stem cells emerge independent of mesodermal influence in vivo, although these experiments do not rule out a possible role for visceral endoderm effects in vivo.

ES-derived primitive neural stem cell spheres express some nonneural marker genes such as GATA4 (an early primitive and definitive endodermal marker; Tropepe et al. 2001), in addition to neural precursor markers such as FGF5 and Sox2 and neural marker Sox1 (Fig. 2B). FGF5 is expressed in primitive ectoderm but down-regulated after E7.5 in the mouse embryo (Hébert et al. 1991), whereas Sox1 expression is first detectable in the neural plate at E7.5 of the mouse embryo, and Sox2 is expressed earlier in the epiblast (Wood and Episkopou 1999). These gene expression properties of ES cell-derived spheres are unaltered after repeated passaging procedures (Fig. 2B). RT-PCR was used to analyze marker gene expression in the primary LIF-dependent and passaged EGF-dependent spheres from the E6.5 mouse epiblast, a time when mesoderm does not underlie the anterior epiblast. Primary E6.5 epiblast-derived spheres showed a gene expression pattern very similar to that of ES spheres; they expressed GATA4, FGF5, Sox2, and Sox1 (Fig. 2B), but did not express Brachyury (a mesodermal marker), HNF4 (a mature endodermal marker), or cytokeratin17 (a epidermal marker). In contrast, E6.5 spheres that were passaged in EGF down-regulated GATA4 and FGF5 expression and presented a similar gene expression pattern to that of definitive neural stem cell neurospheres isolated from the later embryonic brain (Fig. 2B). These results suggest that epiblast-derived LIF-dependent spheres arise from 

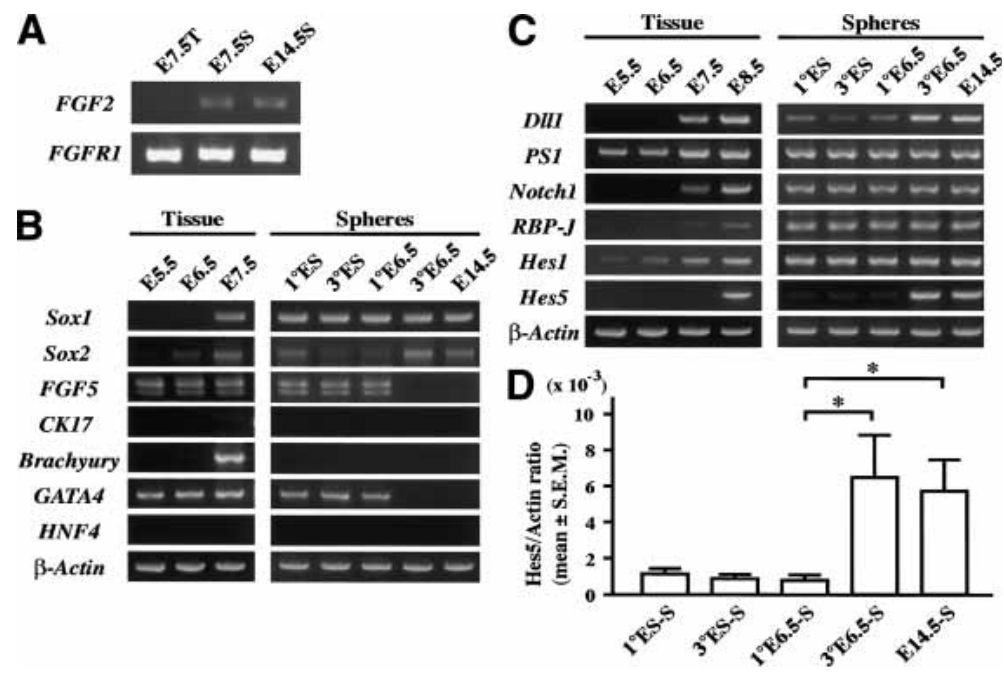

Figure 2. Gene expression profiles of the primary tissue and clonal spheres. $(A)$ Primary E7.5 neuroectoderm-derived spheres (E7.5S), tissue (E7.5T) from which the sphere derived, and E14.5 definitive neural stem cell neurospheres (E14.5S) were analyzed by RT-PCR for the expression of FGF2 and FGF receptor 1 (FGFR1). $(B, C)$ Primary and tertiary E6.5 epiblast-derived spheres $\left(1^{\circ}\right.$ and $3^{\circ}$ E6.5), as well as ES-derived primitive neural stem cell spheres $\left(1^{\circ}\right.$ and $\left.3^{\circ} \mathrm{ES}\right)$ and primary E14.5 definitive neural stem cell spheres (E14.5) were analyzed by RT-PCR. (D) The amounts of Hes5 gene mRNA were quantified by real time RT-PCR using the LightCycler system. The ratios of Hes5 mRNA copy numbers to those of $\beta$-actin are shown. Data represent means \pm S.E.M. $\left(^{\star}\right) P<0.05$.

the proliferation of a neural stem cell equivalent to the ES cell-derived primitive neural stem cells. However, the in vivo E6.5 primitive neural stem cells differentiate in vitro into neural stem cells that are similar to those derived from the forebrains of late embryos or adult mice, unlike the ES-derived primitive neural stem cells whose gene expression profiles do not mature to resemble definitive neural stem cells and do not produce EGF-responsive definitive neural stem cells.

Notch signaling is essential for the maintenance of the neural stem cell, by enhancing the self-renewal of the neural stem cell and by inhibiting its differentiation into neuronal and glial progenitors (Nakamura et al. 2000; Hitoshi et al. 2002). The generation of primary ES spheres in vitro was unaltered in ES cells deficient for the common Notch downstream signaling factor RBP-J (Hitoshi et al. 2002), suggesting that the formation of primitive neural stem cells is independent of Notch activity. However, the role of Notch signaling on the generation of primitive neural stem cells in vivo remains unknown. Expression profiles for Notch pathway genes from the tissues of early embryos, from which spheres were generated, and from the clonal epiblast spheres themselves, were analyzed by RT-PCR (Fig. 2C). Notch activation was assessed by the extent to which Hes5, one of the downstream target genes of Notch signaling, was expressed (de la Pompa et al. 1997; Donoviel et al. 1999; Handler et al. 2000). Hes5 expression was first detected in the neuroepithelium at E8.5, when definitive FGF2dependent neural stem cells first appear in vivo. The other molecules in the Notch pathway were expressed stably in the primary and passaged ES-derived and E6.5 epiblast-derived primitive neural stem cell spheres, as well as in E14.5 definitive neural stem cell neurospheres (Fig. 2C). However, Hes5 expression (assessed by quantitative RT-PCR) in the LIF-dependent, primary E6.5 spheres, as well as in the primary ES spheres, was much weaker (and sometimes below detectable levels) compared with the substantial expression in E14.5 definitive neurospheres (Fig. 2C,D). After passaging in vitro, FGF2- or EGF-responsive tertiary E6.5 spheres up-regulated the Hes5 gene to an extent similar to that observed in the E14.5 brain-derived neurospheres. In contrast, passaged ES spheres (which self-renew in the presence of LIF and FGF2 but never in the presence of EGF) continued to express only weakly (if at all) the Hes5 gene (Fig. 2C,D). These results suggest that Notch signaling is activated strongly during the transition from primitive to definitive neural stem cells in vitro and in vivo.

The in vivo roles of Notch signaling in the generation of primitive and definitive neural stem cells were further investigated using mouse embryos deficient for Notch1 (Conlon et al. 1995). Comparable numbers of LIF-dependent primitive neural stem cells were present in the neural plate of E7.5 Notch1 $1^{-/}$embryos and their littermate controls (Fig. 3A). The primary LIF-dependent spheres from E7.5 Notch1 $^{-1-}$ embryos expressed less Hes5 than their littermate controls (Fig. 3B), but still retained neural multipotentiality (Fig. 3C). On the other hand, the FGF2-responsive definitive neural stem cells in the E8.0 Notch1 $1^{-1-} \mathrm{em}$ bryos were significantly lower in number than those in their littermate controls (Fig. 3D). This decrement was not due to a delayed transition in the Notch $1^{-1-}$ embryos from primitive to definitive neural stem cells, because LIF-dependent primitive neural stem cell numbers decreased similarly between E7.5 and E8.0 in Notch1 ${ }^{-1}$ and control embryos (Fig. 3A). The number of tertiary EGF-responsive definitive neural stem cells generated by passaging primary E7.5 LIF-dependent primitive neural stem cell spheres in vitro was reduced greatly in Notch $1^{-1-}$ embryos compared with their littermate controls (Fig. 3E). Thus, the Notch1 mutation impaired the transition from the primitive to definitive neural stem cells not only in vivo (Fig. 3A,D), but also in vitro (Fig. 3E).

These observations prompted us to ask if Notch activation promotes the transition from the LIF-dependent ES cell-derived primitive neural stem cell to the EGFdependent neural stem cell. ES cell-derived spheres, which can be maintained continuously in LIF and FGF2 media but not in EGF media, were infected with retrovirus expressing constitutively active Notch 1 or control retrovirus (both express enhanced GFP as a reporter; Fig. $4 \mathrm{~A})$, and then cultured in LIF and FGF2 media to generate secondary ES spheres. The resultant individual secondary ES spheres showed variable GFP expression patterns, because infection and retroviral gene integration may occur at different times during sphere formation. Strongly $\mathrm{GFP}^{+}$secondary ES spheres were selected under a fluorescent microscope and passaged to yield tertiary ES spheres in LIF and FGF2 or in EGF alone. Significant numbers of EGF-responsive tertiary ES spheres were observed only after infection with active Notch1 retrovirus (Fig. 4B), whereas very few EGF-responsive tertiary ES spheres were found after control retrovirus infection. The numbers of tertiary ES spheres grown in both LIF 
A

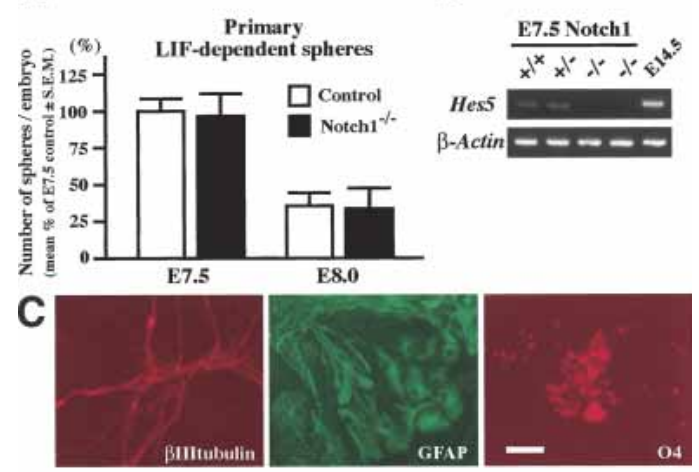

D

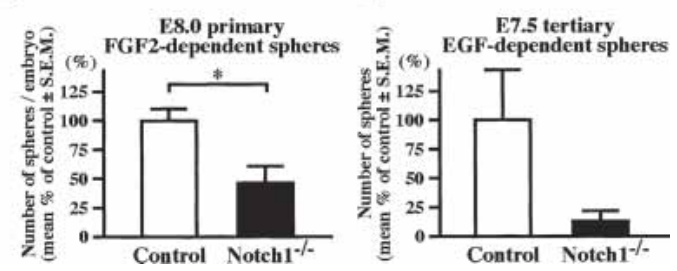

Figure 3. The formation of neural stem cell spheres from Notch1 mutant embryos. (A) The numbers of primary LIF-dependent spheres from E7.5 and E8.0 Notch1 ${ }^{-1-}$ embryos $\mid n=7$ at E7.5 and $n=6$ at E8.0) and littermate controls $(n=18$ at E7.5 and $n=25$ at E8.0) are shown. (B) The expression of Hes5 was abolished in the primary E7.5 Notch $^{-1-}$ spheres. (C) The primary E7.5 Notch1 $1^{-1-}$ spheres retained multipotentiality within the neural lineage. Bar, 50 $\mu \mathrm{m}$. (D) The numbers of primary FGF2-dependent neurospheres from E8.0 Notch1 ${ }^{-/}$embryos $(n=19)$ and littermate controls $(n=57) .\left(^{\star}\right) P<0.05$. (E) The primary LIF-dependent spheres from each E7.5 Notch1 $1^{-1-}(n=7)$ and littermate control $(n=18)$ embryo were separately passaged twice in vitro to generate tertiary EGFdependent neurospheres. The numbers of EGF-dependent tertiary neurospheres from each embryo are shown.

and FGF2 were comparable between the ES spheres infected with active Notch 1 and with control retrovirus, demonstrating that cell numbers plated and viability of the cells were comparable between both groups. Notch signaling was activated, as assessed by the greater amounts of Hes5 gene expression in the tertiary EGFresponsive spheres infected with the active Notch 1 retrovirus than in similar spheres infected with the control retrovirus (Fig. 4C). These results demonstrated that activation of the Notch pathway promotes the emergence of EGF-responsive spheres from LIF-dependent ES spheres. Thus, both in vivo data using the Notch1-/- embryos and in vitro experiments using the ES cell-derived spheres suggest that Notch activation is important for the transition from the LIF-dependent primitive neural stem cell to the EGF-dependent definitive neural stem cell and for the maintenance of the definitive neural stem cell state.

Early experiments using animal cap explants (that are uncommitted ectoderm and normally give rise to epidermis) from Xenopus blastula-stage embryos revealed that after dissociation at low density, the cells became neural tissue (Godsave and Slack 1989; Grunz and Tacke 1989; Sato and Sargent 1989). Later, this fating to neural cells was shown to be accomplished by the inhibition of TGF- $\beta$ activity (Smith et al. 1993; Hemmati-Brivanlou and Melton 1994; Sasai et al. 1995), leading to the pro- posal that neural fate specification from uncommitted blastocyst tissue occurs by a default mechanism. We have shown that this default model may be applicable to mammalian neural induction by demonstrating that mouse ES cells cultured at low cell density (or even as single cells) in serum-free media took on neural identities after $4 \mathrm{~h}$ (Tropepe et al. 2001). In the presence of LIF, a small percentage of the ES cells that may default to a neural state proliferated as primitive neural stem cells in vitro (Tropepe et al. 2001). We suggest that in vivo, where cell density and cell-to-cell interactions are high, pluripotent inner cell mass and epiblast cells receive dense signals that inhibit neural induction and the cells may acquire minimal neural identities as primitive neural stem cells (or at least as potential primitive neural stem cells in vivo), until neural inhibition is fully suppressed by TGF- $\beta$ family antagonists at E7.5-E8.5 when definitive neural stem cells are formed.

Only a few cells that reside in the anterior part of E7.5 neuroectoderm formed clonal primitive neural stem cell spheres $(-0.1 \%)$. The isolation of only rare primitive neural stem cells might be explained in three ways. First, most (if not all) of the putative neuroectodermal cells possess the potential to respond to LIF to proliferate and form spheres, but many may be unable to survive the trituration and serum-free culture conditions. Second, only a few specific cells among all of the putative neuroectoderm cells retain (or can take on) a neural stem cell fate. It would be intriguing to use factors that enhance the survival of (primitive) neural stem cells in the serumfree sphere assay. Indeed, we have found some survival factors increase the cell viability of ES-derived neural cells so that much higher frequencies of spheres are formed (Smukler et al. 2003). However, these same survival factors appear not to increase the frequency of isolating primitive neural stem cells from the epiblast (S. Hitoshi, unpubl.), suggesting that only a few epiblast/ early neuroectoderm cells may have the potential to be
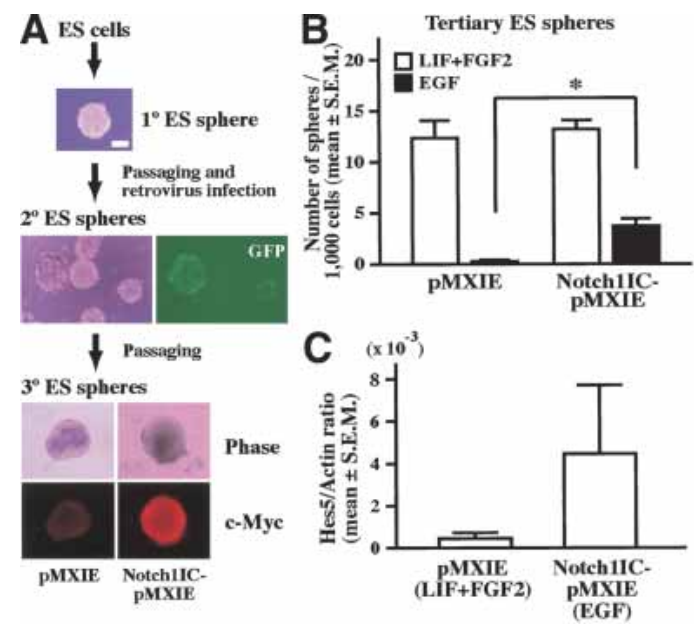

Figure 4. Active Notch1 retrovirus infection of ES spheres. (A) A schema of the experimental procedures. Some of secondary spheres infected with the retrovirus showed $\mathrm{GFP}^{+}$expression. After passaging, tertiary spheres infected with pMXIE-Notch1IC were homogenously positive for c-Myc that is tagged to Notch1IC. Bar, $0.1 \mathrm{~mm}$. $(B)$ The numbers of tertiary spheres generated in both LIF and FGF2 and in EGF-only media are shown. Data represent means \pm S.E.M. from three independent experiments. $\left(^{\star}\right) P<0.05$. (C) The amounts of Hes5 gene expression were quantified $(n=3)$. 
primitive neural stem cells. Third, the rarity of primitive neural stem cells in the epiblast/neuroectoderm might derive from the presence of a few pluripotent ES cells that remain until E7.5 of mouse development. However, this third possibility seems unlikely because ES cells never have been isolated from mouse embryos after the egg cylinder stages (Smith 2001) and because the number of sphere-forming primitive neural stem cells increased during E5.5-E7.0 in the epiblast/neuroectoderm (present study).

In the mouse embryo deficient for Notch pathway molecules, the size of the definitive neural stem cell pool is reduced (Nakamura et al. 2000; Hitoshi et al. 2002). Activation of Notch signaling is indispensable for maintaining the neural stem cell by enhancing its self-renewal capacity and by repressing differentiation into progenitor cells (Fig. 5; Hitoshi et al. 2002). The current results suggest an additional role for Notch signaling in neural stem cell ontogenesis; activation of Notch pathway is required for the transition from the primitive neural stem cell to the definitive neural stem cell, which subsequently acquires EGF responsiveness. The reduction of definitive neural stem cells observed in E8.0 Notch $1^{-1-}$ embryos in vivo, as well as the reduction seen in the EGF-responsive tertiary spheres from E7.5 Notch $1^{-/-}$primitive neural stem cell spheres in vitro, are consistent with this model. Certainly, the appearance of some definitive neural stem cells in the E7.5 Notch $^{-1-}$ embryos suggests that other signaling pathways may permit some transition from primitive to definitive neural stem cells. However, another model also is possible: definitive neural stem cells are generated from the primitive neural stem cells independent of Notch signaling, but definitive neural stem cells may require Notch sig-

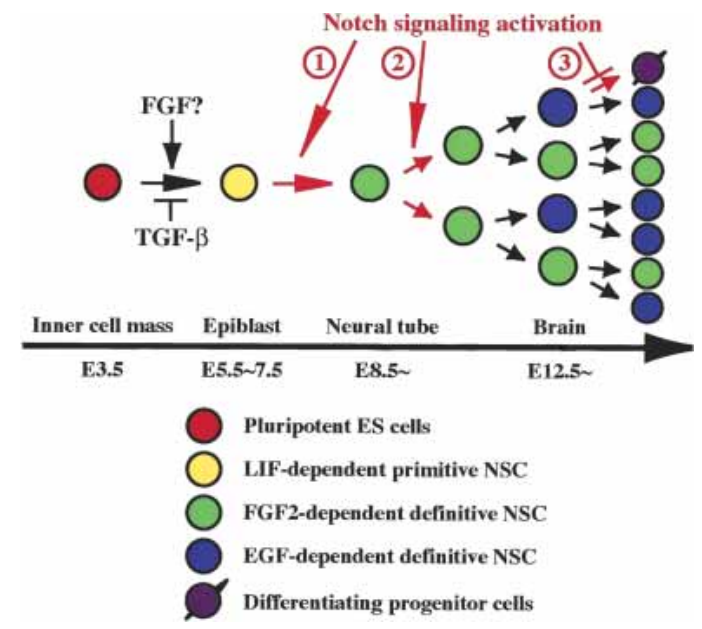

Figure 5. A model of neural stem cell development. LIF-dependent primitive neural stem cells can be generated directly from single ES cell in vitro after relieving TGF- $\beta$ inhibition (Tropepe et al. 2001) and now have been shown to exist (at least potentially in vivo and literally in vitro) in the E5.5-E7.5 epiblast/neuroectoderm of mouse embryos. Whether or not FGF signaling promotes the induction of primitive neural stem cells in vivo (or just their survival or proliferation) remains to be determined. Activation of the Notch pathway could be required for the transition from primitive to definitive neural stem cells that autonomously acquire EGF responsiveness (1) and/or for the maintenance of definitive, FGF2- or EGF-dependent neural stem cells by enhancing their self-renewal (2) and thus suppressing their differentiation into neuronal or glial unipotential progenitors (3). naling for their maintenance, as suggested previously (Hitoshi et al. 2002). These two possibilities are not mutually exclusive and current data do not allow us to discriminate between them. Later in development, Notch signaling may play additional roles in enhancing the symmetric and self-renewing divisions of definitive neural stem cells and suppressing asymmetric division of neural stem cells to produce neuronal and glial progenitor cells (Hitoshi et al. 2002). This later function appears inconsistent with the recent notion of an instructive role for Notch signaling to produce glia from neural progenitor cells in the mammalian central nervous system /Gaiano et al. 2000). However, this inconsistency disappears if adult forebrain neural stem cells acquire some glial features (but do not differentiate into unipotential glial cells), as suggested by demonstrations that Notch signaling enhances GFAP transcription in adult neural progenitor cells (Tanigaki et al. 2001) and that at least some of the GFAP-expressing astrocytes in the adult forebrain subependyma are, indeed, neural stem cells (Doetsch et al. 1999; Morshead et al. 2003).

\section{Materials and methods}

\section{Animals and cell culture}

CD1 mice (Charles River) were used in this study. The generation and genotyping of Notch1 mutant mice (Conlon et al. 1995) and GFP transgenic mice (Hadjantonakis et al. 1998) on a CD1 background have been described. Midday of the plugged day was termed E0.5 and the staging of the early embryos followed (Downs and Davies 1993). Prospective head regions from E7.5 mouse embryos at late primitive streak stage or at early headfold stage, or from E8.0-E8.5 embryos at late headfold stage but before turning were excised and treated with papain $(1 \mathrm{unit} / \mathrm{mL}$, Worthington) in phosphate-buffered saline containing $0.2 \mathrm{mg} / \mathrm{mL}$ cysteine, 5 $\mathrm{mg} / \mathrm{mL}$ glucose, and $0.4 \mathrm{mg} / \mathrm{mL}$ bovine serum albumin for $4 \mathrm{~min}$. Neuroepithelium was detached from underlying tissue and triturated into single cells. Usually, 2000-3000 viable cells were collected from each E7.5 embryo. The cells were cultured in serum-free media (Tropepe et al. 1999) containing LIF $\left(1 \times 10^{3}\right.$ units $/ \mathrm{mL}$, Chemicon) and B27 supplement (Invitrogen). The cells from each embryo at E7.5 or E8.0 were cultured in 24-well $(0.5 \mathrm{~mL} /$ well) plates, where usual cell densities were below 10 cells $/ \mu \mathrm{L}$. The anterior parts of the E6.5-E7.0 embryos at the early primitive streak stage were excised, triturated into single cells, and cultured as described earlier. Distal portions of the E5.5-E6.5 embryos at the egg cylinder stage or at the preprimitive streak stage were also excised, roughly triturated leaving small clumps, and then cultured as described earlier. The dissection and cell culture of forebrains from mouse embryos at and after E8.5 or adult mice, the passaging procedures, differentiation assay, and immunohistochemical analysis procedures also have been described (Tropepe et al. 1999; Hitoshi et al. 2002).

\section{Immunocytochemistry}

Immunocytochemical analyses were performed as described previously (Hitoshi et al. 2002). We used anti-nestin mouse monoclonal (IgG; 1:100, Chemicon), anti- $\beta$ III tubulin mouse monoclonal (IgG; 1:200; Sigma), anti-GFAP rabbit polyclonal (IgG; 1:400; Chemicon), anti-O4 mouse monoclonal (IgM; 1:40; Roche), or anti-c-Myc mouse monoclonal (IgG; 1:500; Santa Cruz) antibodies as primary, followed by appropriate FITCor TRITC-conjugated secondary antibodies. Cultures were counterlabeled with the nuclear stain Hoechst $33258(1 \mathrm{\mu g} / \mathrm{mL}$; Sigma).

RT-PCR

cDNA synthesis, some of the primer sequences, and PCR cycling procedures have been described (Tropepe et al. 2001; Hitoshi et al. 2002). Primer sequences and PCR cycling conditions will be provided on request. Quantitative RT-PCR analyses for Hes 5 and $\beta$-actin were performed using the LightCycler system (Roche) and using the same procedures described previously (Takahashi et al. 2003).

Retrovirus infection

The construction of replication-incompetent retroviral vector, pMXIE and NotchlIC-pMXIE, retrovirus preparation, and infection procedures 
have been described (Hitoshi et al. 2002). Primary ES spheres were dissociated, infected with retrovirus, and then cultured in LIF and FGF2 media to generate secondary ES spheres. The resultant individual secondary ES spheres showed variable GFP expression patterns, because infection and retroviral gene integration may occur at different times during sphere formation. Strongly $\mathrm{GFP}^{+}$secondary ES spheres were picked up under a fluorescent microscope and passaged to yield tertiary ES spheres.

\section{Acknowledgments}

We thank R. Conlon for the Notch1 mutant mice and J. Nye for Nterminal Myc epitope-tagged Notch1IC. This work was supported by grants from the Canadian Institutes of Health Research (D.v.d.K.), and Grants-in-Aid for Scientific Research on Priority Areas-Advanced Brain Science Project-from the Ministry of Education, Culture, Sports, Science and Technology of Japan (S.H.), "Research for the Future (RFTF)" of the Japan Society for the Promotion of Science (S.H.), and the Cell Science Foundation (S.H.).

The publication costs of this article were defrayed in part by payment of page charges. This article must therefore be hereby marked "advertisement" in accordance with 18 USC section 1734 solely to indicate this fact.

\section{References}

Artavanis-Tsakonas, S., Matsuno, K., and Fortini, M.E. 1995. Notch signaling. Science 268: 225-232.

Conlon, R.A., Reaume, A.G., and Rossant, J. 1995. Notch1 is required for the coordinate segmentation of somites. Development 121: 15331545.

de la Pompa, J.L., Wakeham, A., Correia, K.M., Samper, E., Brown, S., Aguilera, R.J., Nakano, T., Honjo, T., Mak, T.W., Rossant, J., et al. 1997. Conservation of the Notch signalling pathway in mammalian neurogenesis. Development 124: 1139-1148.

Doetsch, F., Caille, I., Lim, D.A., Garcia-Verdugo, J.M., and AlvarezBuylla, A. 1999. Subventricular zone astrocytes are neural stem cells in the adult mammalian brain. Cell 97: 703-716.

Donoviel, D.B., Hadjantonakis, A.-K., Ikeda, M., Zheng, H., St George Hyslop, P., and Bernstein, A. 1999. Mice lacking both presenilin genes exhibit early embryonic patterning defects. Genes \& Dev. 13: 2801-2810.

Downs, K.M. and Davies, T. 1993. Staging of gastrulating mouse embryos by morphological landmarks in the dissecting microscope. Development 118: 1255-1266.

Gaiano, N., Nye, J.S., and Fishell, G. 2000. Radial glial identity is promoted by Notch 1 signaling in the murine forebrain. Neuron 26: 395 404.

Godsave, S.F. and Slack, J.M. 1989. Clonal analysis of mesoderm induction in Xenopus laevis. Dev. Biol. 134: 486-490.

Grunz, H. and Tacke, L. 1989. Neural differentiation of Xenopus laevis ectoderm takes place after disaggregation and delayed reaggregation without inducer. Cell Differ. Dev. 28: 211-217.

Hadjantonakis, A.-K., Gertsenstein, M., Ikawa, M., and Nagy, A. 1998. Generating green fluorescent mice by germline transmission of green fluorescent ES cells. Mech. Dev. 76: 79-90.

Handler, M., Yang, X., and Shen, J. 2000. Presenilin-1 regulates neuronal differentiation during neurogenesis. Development 127: 2593-2606.

Hébert, J.M., Boyle, M., and Martin G.R. 1991. mRNA localization studies suggest that murine FGF-5 plays a role in gastrulation. Development 112: 407-415.

Hemmati-Brivanlou, A. and Melton, D.A. 1994. Inhibition of activin receptor signaling promotes neuralization in Xenopus. Cell 77: 273281.

Hitoshi, S., Alexson, T., Tropepe, V., Donoviel, D., Elia, A.J., Nye, J.S., Conlon, R.A., Mak, T.W., Bernstein, A., and van der Kooy, D. 2002. Notch pathway molecules are essential for the maintenance, but not the generation, of mammalian neural stem cells. Genes \& Dev. 16: 846-858.

Kawaguchi, A., Miyata, T., Sawamoto, K., Takashita, N., Murayama, A., Akamatsu, W., Ogawa, M., Okabe, M., Tano, Y., Goldman, S.A., et al. 2001. Nestin-EGFP transgenic mice: Visualization of the self-re- newal and multipotency of CNS stem cells. Mol. Cell. Neurosci. 17: $259-273$.

Kimble, J. and Simpson, P. 1997. The LIN-12/Notch signaling pathway and its regulation. Annu. Rev. Cell Dev. Biol. 13: 333-361.

Morshead, C.M., Garcia, A.D., Sofroniew, M.V., and van der Kooy, D. 2003. The ablation of glial fibrillary acidic protein-positive cells from the adult central nervous system results in the loss of forebrain neural stem cells but not retinal stem cells. Eur. J. Neurosci. 18: 76-84.

Nakamura, Y., Sakakibara, S.-i., Miyata, T., Ogawa, M., Shimazaki, T. Weiss, S., Kageyama, R., and Okano, H. 2000. The bHLH gene Hes 1 as a repressor of the neuronal commitment of CNS stem cells. $J$. Neurosci. 20: 283-293.

Sasai, Y., Lu, B., Steinbeisser, H., and De Robertis, E.M. 1995. Regulation of neural induction by the Chd and Bmp-4 antagonistic patterning signals in Xenopus. Nature 376: 333-336.

Sato, S.M. and Sargent, T.D. 1989. Development of neural inducing capacity in dissociated Xenopus embryos. Dev. Biol. 134: 263-266.

Smith, A. 2001. Embryonic stem cells. In Stem cell biology (eds. D.R. Marshak, R.L. Gardner, and D. Gottlieb), pp. 205-230. Cold Spring Harbor Laboratory Press, Cold Spring Harbor, NY.

Smith, W.C., Knecht, A.K., Wu, M., and Harland, R.M. 1993. Secreted noggin protein mimics the Spemann organizer in dorsalizing Xenopus mesoderm. Nature 361: 547-549.

Smukler, S.R., Xu, S., and van der Kooy, D. 2003. Enhanced ES-derived neurosphere formation with increased cell viability supports a model of default differentiation of ES cells into primitive neural stem cells. Abstr. Soc. Neurosci. 124.6.

Streit, A., Berliner, A., Papanayotou, C., Sirulnik, A., and Stern, C.D. 2000. Initiation of neural induction by FGF signalling before gastrulation. Nature 406: 74-78.

Takahashi, Y., Jeong, S.Y., Ogata, K., Goto, J., Hashida, H., Isahara, K., Uchiyama, Y., and Kanazawa, I. 2003. Human skeletal muscle calcium channel $\alpha 1 S$ is expressed in the basal ganglia: Distinctive expression pattern among L-type $\mathrm{Ca}^{2+}$ channels. Neurosci. Res. 45: 129 137.

Tanigaki, K., Nogaki, F., Takahashi, J., Tashiro, K., Kurooka, H., and Honjo, T. 2001. Notch1 and Notch3 instructively restrict bFGF-responsive multipotent neural progenitor cells to an astroglial fate. Neuron 29: 45-55.

Tropepe, V., Sibilia, M., Ciruna, B.G., Rossant, J., Wagner, E.F., and van der Kooy, D. 1999. Distinct neural stem cells proliferate in response to EGF and FGF in the developing mouse telencephalon. Dev. Biol. 208: 166-188.

Tropepe, V., Hitoshi, S., Sirard, C., Mak, T.W., Rossant, J., and van der Kooy, D. 2001. Direct neural fate specification from embryonic stem cells: A primitive mammalian neural stem cell stage acquired through a default mechanism. Neuron 30: 65-78.

Wilson, S.I. and Edlund, T. 2001. Neural induction: Toward a unifying mechanism. Nat. Neurosci. 4 (Suppl.): 1161-1168.

Wood, H.B. and Episkopou, V. 1999. Comparative expression of the mouse Sox1, Sox2 and Sox3 genes from pre-gastrulation to early somite stages. Mech. Dev. 86: 197-201.

Ying, Q.-L., Stavridis, M., Griffiths, D., Li, M., and Smith, A. 2003. Conversion of embryonic stem cells into neuroectodermal precursors in adherent monoculture. Nat. Biotechnol. 21: 183-186. 


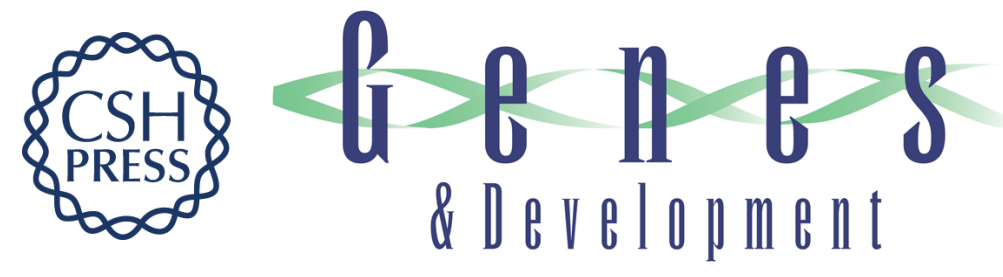

\section{Primitive neural stem cells from the mammalian epiblast differentiate to definitive neural stem cells under the control of Notch signaling}

Seiji Hitoshi, Raewyn M. Seaberg, Cheryl Koscik, et al.

Genes Dev. 2004, 18:

Access the most recent version at doi:10.1101/gad.1208404

References This article cites 28 articles, 9 of which can be accessed free at: http://genesdev.cshlp.org/content/18/15/1806.full.html\#ref-list-1

License

Email Alerting

Receive free email alerts when new articles cite this article - sign up in the box at the top Service right corner of the article or click here.

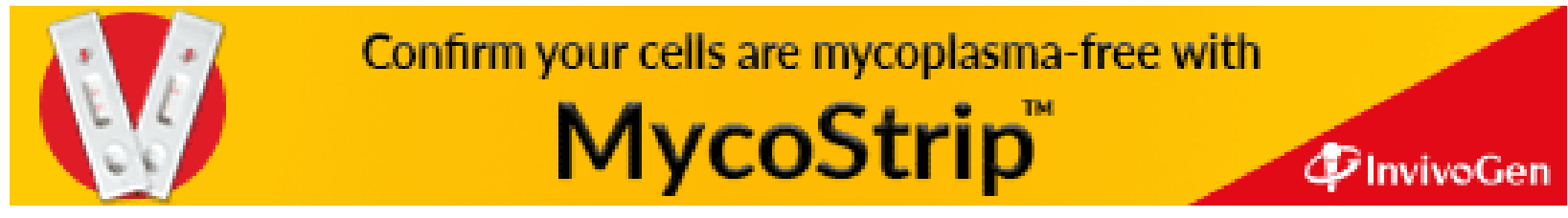

